\title{
Cryo-Electron Microscopy of Highly Condensed DNA inside Virus Capsids
}

\author{
A. C. Steven*, N. Cheng ${ }^{*}$, B. Mai ${ }^{* *}$, A. Jones*, C. Butan*, M.E. Cerritelli ${ }^{*}$, L.W. Black ${ }^{* *}$ \\ * Laboratory of Structural Biology, NIAMS-NIH, Bethesda, MD 20892 \\ ** Department of Biochemistry and Molecular Biology, University of Maryland School of \\ Medicine, Baltimore, MD 21201
}

The replication cycles of most organisms are accompanied by condensation and decondensation of their chromosomes. The difference in compaction between the condensed and decondensed states is extreme in the case of double-stranded DNA viruses, whose encapsidated genomes approach the remarkable packing density of $450 \mathrm{mg} / \mathrm{ml}$. To achieve this state, the DNA is translocated into the capsid by a powerful motor protein, terminase. There is longstanding interest in the packing geometry of encapsidated viral DNA, and cryo-electron microscopy (cryo-EM) provides an incisive approach to studying its organization. Micrographs of DNA-containing heads of bacteriophage T7 viewed in two defined orientations - side views and axial views (defined as such relative to the portal vertex through which DNA enters and exits the capsid) - established the "coaxial spool" model whereby the DNA is coiled around the portal axis in up to six concentric shells [1]. This presentation will summarize cryo-EM observations on packaged DNA in other viruses, in studies intended to explore how general the T7 paradigm may be.

The main focus will be on the configuration of packaged DNA in bacteriophage T4 - incidentally, one of the first viruses to be investigated by cryo-EM [2]. T4 represents a considerably higher degree of complexity than T7 in that its $162 \mathrm{kbp}$ genome is four-fold larger than that of T7 and its capsid is a prolate, not an isometric, icosahedron. Our strategy has been to devise ways to orient phage heads in solution to that they preferentially present either side views or axial views. We also perturbed the packaged DNA by heating it and by digesting it in situ by calcium activation of Staphylococcal nuclease that had been incorporated into the precursor procapsid during its assembly, prior to DNA packaging [3]. For comparison, we also studied mutant T4 heads that are either smaller than normal (isometric) - Figure 1 - or longer than normal (giant), and gained clues about likely configurations of DNA at intermediate stages of packaging from images of underpackaged capsids. To interpret the resulting images, we performed computer modelling.

We conclude that, despite some stochastic variability from particle to particle, the coaxial spool model also applies to T4, with coiling around the portal axis in isometric heads (Fig. 1); perpendicular to it in giant heads; and at intermediate orientations in wild-type prolate heads. While the outer shells are well ordered, there appears to be a region of less well ordered DNA at the center. These observations are consistent with minimization of energy associated with DNA bending being an important factor in determining the geometry of densely packed viral DNA.

\section{References}

[1] M. E. Cerritelli et al., Cell 91 (1997) 271.

[2] J. Lepault et al. EMBO J. 6 (1987) 1507.

[3] J. Mullaney \& L. W. Black, J. Mol. Biol. 283 (1998) 913.

[4] K. Iwasaki et al., Virology 271 (2000) 321. 


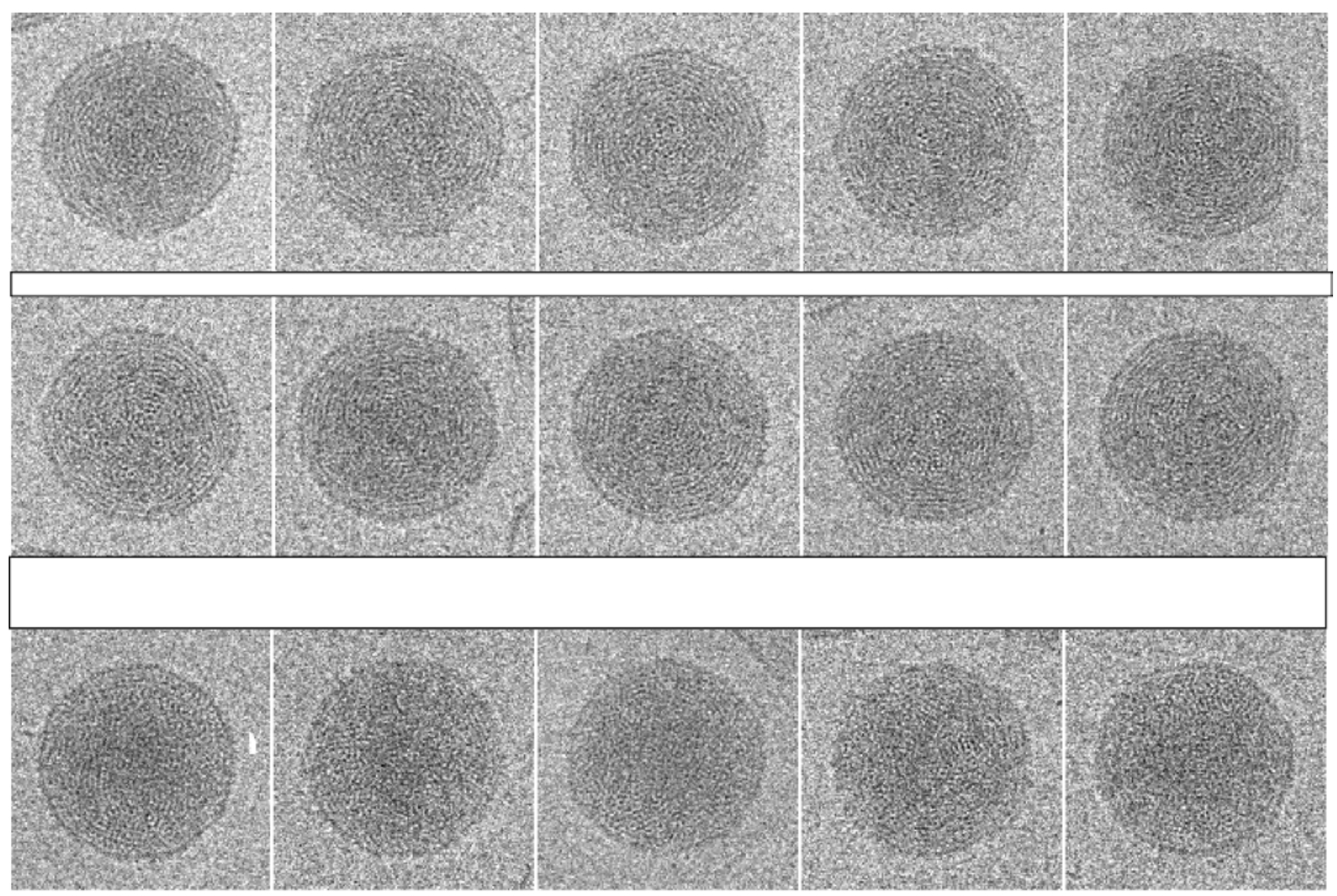

Figure 1. Cryo-electron micrographs of tail-less DNA-filled heads of bacteriophage T4 viewed along axes of five-fold symmetry of the icosahedral capsid shell. Viewed from this direction, the capsid, which has relatively flat facets, looks nearly round in projection. The small serrations around the outer edge of the capsid are contributed by molecules of Hoc, a $40-\mathrm{kDa}$ accessory protein that binds to the mature capsid [4]. The top two rows represent heads that show concentric ring patterns ("fingerprint" motifs). We interpret these heads as being viewed along the portal axis. The heads in the bottom row are also round and therefore also viewed along a 5-fold symmetry axis. They show punctate patterns with the same characteristic spacing of $\sim 25 \AA$. We interpret them as being viewed along one of the other five axes of 5-fold symmetry. 